Rev. Elev. Méd. vét. Pays trop., 1973, 26 (1): 43-53

\title{
Une membrane synthétique utilisée pour la nourriture artificielle des Glossines (Diptera-Muscida)
}

\author{
par J. PAGOT (*), J. ITARD (*) et M. CHOMAT (**) \\ (avec la collaboration technique de Mme C. DEDEVENS)
}

\begin{abstract}
RESUME
Des essais de nourriture artificielle des glossines à travers une membrane synthétique ont été effectués. Les résultats obtenus montrent que des membranes de silicone de $10 / 100 \mathrm{de} \mathrm{mm}$ d'épaisseur permettent de nourrir ces insectes de façon artificielle. Ces membranes sont chimiquement inertes et les qualités physiques des silicones permettent de les stériliser à des températures élevées et de les conserver indéfiniment. La nourriture avec du sang de mouton citraté glucosé, comparativement à la nourriture sur sang défibriné, améliore à la fois la longévité des femelles, leur capacité reproductrice et le poids des pupes produites, lesquels toutefois restent inférieurs aux résultats obtenus chez les témoins nourris sur lapin.
\end{abstract}

\section{INTRODUCTION}

L'élevage des mouches tsé-tsé, nourries quotidiennement sur animal vivant et maintenues, au laboratoire, dans des conditions de température et d'humidité convenables, a pu, depuis quelques années, être réalisé, dans deux ou trois centres, avec des effectifs comportant plusieurs milliers d'individus.

Les résultats obtenus sont tels qu'ils permettent d'envisager la création, en Afrique, d'unités d'élevage de rendement élevé, destinées à appliquer, aux glossines, la méthode de lutte par lâchers de mâles stériles, méthode qui a été utilisée, avec succès, aux U.S.A., contre Cochliomyia hominivorax.

Cependant, l'hématophagie stricte des glossines nécessite que soit réalisé, parallèlement à l'élevage des mouches, un élevage important d'animaux (cobayes, lapins ou chèvres) sur lesquels les insectes seront nourris.

(*) I.E.M.V.T., 10, rue Pierre Curie, 94700 MaisonsAlfort (France).

(**) Société Industrielle des Silicones, Usine de Saint-Fons, quai Louis Aubagne, 69190 Saint-Fons.
La mise au point d'une méthode de nourriture artificielle permettrait d'éviter les servitudes techniques et économiques qu'impose l'élevage de ces animaux.

La nourriture artificielle des glossines, insectes piqueurs hématophages, ne peut être réalisée qu'en offrant, aux insectes, du sang dont on évite la coagulation par des moyens mécaniques ou chimiques. L'insecte ne peut absorber ce sang qu'après avoir percé une membrane dont les caractéristiques physiques rappellent celles du tissu cutané.

Les études sur la nourriture artificielle des glossines doivent donc porter, d'une part sur le type de membrane le plus apte à inciter les mouches à piquer et, d'autre part, sur la composition du liquide nutritif, qui doit se rapprocher le plus possible du sang total de l'animal hôte.

\section{COMPOSITION DE LA MEMBRANE}

Les premières études sur la nourriture artificielle des glossines ont été effectuées, en 1912, 
par RODHAIN et ses collaborateurs, qui constatent que les mouches tsé-tsé absorbent bien le sang à travers une membrane formée par une peau rasée de cobaye ou de souris. Par la suite, différents types de membrane ont été expérimentés : peaux rasées de rat, de lapin, de mouton; intestin d'agneau; peau de saucisson; membrane de fibrine obtenue par précipitation de plasma sanguin par le calcium. La plupart de ces membranes n'ayant pu donner entière satisfaction (réalisation laborieuse, pas d'attirance pour les glossines ou durée de conservation trop brève), les chercheurs anglais ont mis au point une membrane obtenue en versant, sur des couches de gaze ordinaire ou de tulle de térylène, de l'agar dissous dans de l'eau distillée en ébullition, dans la proportion de 3 p. 100. L'épaisseur de cette membrane est de 2 à $2,5 \mathrm{~mm}$. Son principal inconvénient est sa durée de conservation très limitée. L'agar a tendance à se dessécher très rapidement, perdant ainsi sa souplesse, ou bien, si la membrane est conservée en atmosphère humide, elle est envahie dans les 24 heures par des moisissures. Il est par conséquent nécessaire de préparer chaque jour une nouvelle membrane.

C'est la raison pour laquelle les recherches effectuées à l'I.E.M.V.T. se sont orientées vers des produits entièrement artificiels répondant aux critères suivants :

- la membrane obtenue doit pouvoir être traversée par le proboscis des glossines, qui forme un tube capillaire très fin, de $3 \mathrm{~mm}$ de longueur sur moins d'un $\mathrm{mm}$ de largeur moyenne;

- elle doit avoir une élasticité suffisante pour que la solution de continuité créée par la pénétration du proboscis se referme après le retrait de celui-ci;

- elle doit être chimiquement inerte et, en particulier, ne pas contenir de substances chlorées ou de caoutchouc, qui sont toxiques pour les glossines.

Les silicones, dont on connaît la neutralité chimique et les qualités physiques, semblaient pouvoir répondre aux critères désirés.

La Société Industrielle des Silicones (S.I.S.S.), à qui le problème a été soumis, a bien voulu nous fournir des échantillons constitués par des feuilles de $320 \times 320 \mathrm{~mm}$ :
- Silastene $\mathbf{S}$ :

- 2000 de $6 / 10$ de $\mathrm{mm}$ d'épaisseur,

- Silastene RTVS :

- 7037 de $6 / 10$ de $\mathrm{mm}$ d'épaisseur,

- 7037 de 6/100 de mm d'épaisseur,

- 7037 de $10 / 100$ de mm d'épaisseur,

- 7037 de 20/100 de mm d'épaisseur,

- Silastene RTVX :

- 62958 de 10/100 de mm d'épaisseur.

\section{TECHNIQUE}

Le premier objectif étant de vérifier si les insectes acceptaient de se nourrir à travers ces différents types de membrane, il suffisait d'utiliser un appareillage aussi simple que possible, réduisant les manipulations au minimum.

10 à $15 \mathrm{ml}$ de sang de bœuf ou de mouton, citraté ou défibriné, sont versés dans des récipients en verre, d'un modèle courant du commerce, de $120 \mathrm{ml}$ de capacité. Un disque de $105 \mathrm{~mm}$ de diamètre, découpé dans la membrane à tester et maintenu en place par un bracelet de caoutchouc, ferme ces récipients qui sont réchauffés au bain-marie à $37^{\circ} \mathrm{C}$, puis retournés sur les cages contenant les glossines. La pression de l'air chaud à l'intérieur du récipient suffit à donner à la membrane une tension correcte. Les glossines piquent de bas en haut, en s'accrochant à la face supérieure de la cage. Toutes les cinq minutes, les récipients sont à nouveau réchauffés au bainmarie.

Les lots de mouches mis à nourrir chaque jour, à l'exception du dimanche, sur les membranes à tester, ont été suivis pendant 80 jours minimum; les mortalités et le nombre de pupes produites par les femelles ont été relevés, pour chaque lot, et comparés avec les mortalités et productions de pupes de lots témoins de même âge, nourris sur oreilles de lapin.

\section{RESULTATS}

\section{A. Membranes de $6 / 10$, de $6 / 100$ et de $10 / 100$ de $\mathrm{mm}$ d'épaisseur}

Les résultats obtenus à la suite de nombreux essais effectués avec les membranes S 2000 de $6 / 10$, RTVS 7037 de 6/10, RTVS 7037 de $6 / 100$, de $10 / 100$ et de $20 / 100$ et RTVX 62958 de $10 / 100$, ont fait retenir, pour la 
poursuite de l'étude, la membrane RTVS 7037 de 10/100 de mm d'épaisseur, qui s'est révélée être la plus attractive. La durée moyenne de vie $(59,1$ jours $\pm 22,8)$ des individus mâles nourris sur cette membrane est nettement supé- rieure aux durées moyennes de vie des deux autres lots nourris sur membrane, et se rapproche de la durée moyenne de vie du lot témoin $(72,6$ jours $\pm 17,2)$ nourri sur oreille de lapin (tableau I).

TABLEAU $\mathrm{N}^{\circ} \mathrm{I}$

Longévité des mâles témoins et nourris sur membrane

\begin{tabular}{|c|c|c|c|c|c|}
\hline$N^{0}$ & Caractéristique & $\begin{array}{l}\text { Durée moyenne } \\
\text { de vie en } \\
\text { jours (m) }\end{array}$ & Ecart-type (s) & $\begin{array}{c}\text { Coefficient de } \\
\text { variation }(\mathrm{p}, 100) \\
(100 \mathrm{~s} / \mathrm{m})\end{array}$ & $\begin{array}{c}\text { Intervalle de confiance } \\
\text { de la moyenne } \\
\text { théorique pour } \\
\text { a }=5 \text { p.100 }\end{array}$ \\
\hline 1 & Témoins & 72,60 & 27,9256 & 38,3273 & $55,35-89,84$ \\
\hline 2 & $\begin{array}{l}\text { RTVS } 7037 \\
6 / 100\end{array}$ & 22,80 & 24,3392 & 106,7512 & $7,71-37,88$ \\
\hline 3 & $\begin{array}{l}\text { RTVS } 7037 \\
10 / 100\end{array}$ & 59,10 & 36,9247 & 62,4783 & $36,21-81,98$ \\
\hline 4 & $\begin{array}{l}\text { RTVX } 62958 \\
\text { LO/100 }\end{array}$ & 31,90 & 26,7725 & $B 3,9263$ & $15,30-48,49$ \\
\hline $2,3,4$ & $\begin{array}{l}\text { Ensemble des } \\
\text { lots nourris } \\
\text { sur membrane }\end{array}$ & 37,93 & 32,7939 & 86,4516 & $26,19-49,66$ \\
\hline
\end{tabular}

Une autre étude a également été effectuée avec la membrane RTVS 7037 de $20 / 100$ et du sang de mouton défibriné ou citraté glucosé.

\section{B. Membrane RTVS 7037 de $10 / 100$ de $\mathrm{mm}$ d'épaisseur}

Les essais effectués avec cette membrane ont porté sur deux lots de femelles d'espèces différentes, nourries avec du sang défibriné.

Le premier lot a été constitué par 117 femelles de G. austeni nourries sur sang de bovin défibriné, qui a été comparé à un lot témoin de 308 femelles de la même espèce, écloses à la même époque, et nourries sur oreilles de lapin.

Le second lot a été constitué par 120 femelles de $G$. morsitans morsitans nourries sur sang de mouton défibriné, qui a été comparé à un lot témoin de 339 femelles de la même espèce, écloses à la même époque et nourries sur oreilles de lapin.

Dans chaque cas, on a comparé les durées moyennes de survie des femelles d'expérience et des femelles témoins, leur production de pupes, et le poids de ces pupes, pesées dans les 24 heures suivant la ponte.

\section{Longévité des femelles}

La longévité des femelles ( $G$. austeni et G. morsitans) nourries sur oreilles de lapin a largement dépassé 150 jours. La durée moyenne de vie, pour ces deux lots témoins, est d'environ 70 jours.

Les longévités maximales des femelles nourries sur membrane ont atteint 103 jours (G. austeni) et 108 jours (G. morsitans).

Les durées moyennes de vie ont été d'environ 25 jours, pour $G$. austeni et 32 jours, pour $G$. morsitans.

Il n'y a pas de différence significative (tableau II) entre les durées moyennes de vie des lots de femelles nourries sur lapin.

Les durées moyennes de vie des femelles nourries sur membrane sont, par contre, quelle que soit l'espèce, significativement plus courtes que celles des témoins, et la durée moyenne de vie des $G$. austeni nourries sur membrane est significativement plus faible que celle des $G$. morsitans nourries sur membrane.

\section{Production de pupes}

Afin de comparer dans les mêmes conditions les productions de pupes de ces quatre lots, il n'a été tenu compte que des productions obtenues en 80 jours ( 8 décades). Dans les conditions naturelles, une femelle de glossine ne produit qu'une larve à la fois, la première à l'âge de 18-20 jours, et les suivantes à 10 jours d'intervalle, soit, à partir du $20^{\mathrm{e}}$ jour de vie, 
TABLEAU $N^{*}$ II

Longévité des femelles de $G$, austeni et de $G$, morsitans nourries sur lapin et sur membrane $10 / 100$

\begin{tabular}{|c|c|c|c|c|c|c|}
\hline $\mathbb{N}^{\bullet}$ & Caractêristique & $\begin{array}{l}\text { Durée moyenne } \\
\text { de vie en } \\
\text { jours (m) }\end{array}$ & \multicolumn{2}{|c|}{$\begin{array}{c}\text { Ecart-type } \\
(\mathrm{s})\end{array}$} & $\begin{array}{c}\text { Caefficient de } \\
\text { variation }(\mathrm{p}, 100) \\
(100 \mathrm{~s} / \mathrm{m})\end{array}$ & $\begin{array}{c}\text { Intervalle de confiance } \\
\text { de la moyenne } \\
\text { thëorique pour } \\
\alpha=5 \text { p. } 100\end{array}$ \\
\hline 1 & $\begin{array}{l}\text { G. austeni femelles } \\
\text { nourries sur lapin }\end{array}$ & 70,32 & \multicolumn{2}{|c|}{41,4468} & 58,9337 & $65,70-74,95$ \\
\hline 2 & $\begin{array}{l}\text { G. austeni femelles } \\
\text { nourries sur membrane }\end{array}$ & 24,96 & \multicolumn{2}{|c|}{27,3744} & 109,6478 & $20,00-29,92$ \\
\hline 3 & $\begin{array}{l}\text { G. morsitans femelles } \\
\text { nourries sur lapin }\end{array}$ & 71,15 & \multicolumn{2}{|c|}{29,8534} & 41,9558 & $68,05-74,25$ \\
\hline 4 & $\begin{array}{l}\text { G. morsitans femelles } \\
\text { nourrieg sur membrane }\end{array}$ & 31,94 & \multicolumn{2}{|c|}{19,4453} & & $28,46-35,42$ \\
\hline \multicolumn{4}{|c|}{ Comparaison des moyennes entre : } & \multicolumn{2}{|c|}{ Valeur de $\mathrm{E}$} & $\begin{array}{l}\text { Signification pour } \\
a=5 \mathrm{p} \cdot 100\end{array}$ \\
\hline \multirow{3}{*}{\multicolumn{4}{|c|}{$\begin{array}{l}1 \text { et } 3 \text { (G. austeni et } G \text {. morsitans nourrıes sur lapin) } \\
1 \text { et } 2 \text { (G. austeni nourries sur lapin et sur membrane) } \\
3 \text { et } 4 \text { (G. morsitans nourries sur lapin et sur membrane) }\end{array}$}} & \multicolumn{2}{|r|}{0,2907} & N.S. \\
\hline & & & & \multicolumn{2}{|r|}{13,1046} & s. \\
\hline & & & & \multicolumn{2}{|r|}{16,4904} & s. \\
\hline \multicolumn{4}{|c|}{$\begin{array}{c}2 \text { et } 3 \text { (G. austeni nourries sur membrane et } \\
\text { G. morsitans nourries sur lapin) }\end{array}$} & \multicolumn{2}{|r|}{15,4753} & s. \\
\hline \multicolumn{4}{|c|}{$\begin{array}{l}1 \text { et } 4 \text { (G. austeni nourries sur lapin et } \\
\text { G. morsitans nourries sur membrane) }\end{array}$} & \multicolumn{2}{|r|}{12,9929} & s. \\
\hline \multicolumn{4}{|c|}{$\begin{array}{c}2 \text { et } 4 \text { (G. austeni et } G \text {. morsitans nourries sur } \\
\text { membrane) }\end{array}$} & \multicolumn{2}{|r|}{2,2566} & s. \\
\hline
\end{tabular}

une pupe par décade. Dans les tableaux III et IV, on a calculé les longévités et les productions de pupes, par décade, en les ramenant à 100 femelles vivantes au jour 0 .

Les productions totales de pupes obtenues, en 80 jours, pour 100 femelles initiales, sont donc liées à la mortalité des femelles.

Par cette méthode, on obtient, pour 100 femelles initiales, en 80 jours :

- 57 pupes chez $G$. austeni nourrie sur membrane $10 / 100$ et sang défibriné;

- 380 pupes chez $G$. austeni nourrie sur lapin;

- 90 pupes chez G. morsitans nourrie sur membrane $10 / 100$ et sang défibriné;

- 341 pupes chez $G$. morsitans nourrie sur lapin.

Afin d'éliminer le facteur mortalité, le nombre de pupes produites par une femelle, dans chaque décade, a été multiplié par 100, comme s'il $\mathrm{y}$ avait 100 femelles vivantes à chaque décade, et on a effectué, pour chaque lot, la moyenne sur 8 décades. On obtient ainsi les productions moyennes, par décade, pour 100 femelles vivantes, du tableau V.
Les comparaisons de ces moyennes montrent qu'il n'y a pas de différence significative entre les productions des différents lots, malgré les différences des moyennes absolues entre les lots nourris sur lapin et ceux nourris sur membrane. Ce dernier mode de nourriture ne semble donc pas affecter directement les capacités reproductrices des femelles.

\section{Poids des pupes}

Toutes les pupes produites par les femelles nourries sur membrane ont été systématiquement pesées, le jour même de la récolte. Ces poids ont été comparés à ceux de pupes, prises au hasard, de femelles d'élevage des mêmes espèces nourries sur lapin. Les poids moyens de ces différents lots figurent dans le tableau VI.

Les comparaisons des moyennes montrent une différence très significative entre les poids des pupes produites par les femelles nourries sur membrane et ceux des pupes produites par les femelles nourries sur lapin. Ces dernières ont un poids nettement plus élevé que celui des pupes issues de femelles nourries sur membrane. 
TABLEAL $N^{\circ}$ III

Longếvité et production de pupes

\begin{tabular}{|c|c|c|c|c|c|c|}
\hline \multicolumn{7}{|c|}{ Femelles $G$. austen $i$ nourries sur membrane $10 / 100$} \\
\hline Jours & 1 & 2 & 3 & 4 & 5 & 6 \\
\hline 0 & 117,00 & 100,00 & & & & \\
\hline $1-10$ & 80,90 & 69,1452 & & & & \\
\hline $11-20$ & 48,10 & 41,1111 & 3,00 & 0,0623 & 2,5641 & 2,5641 \\
\hline $21-30$ & 41,70 & 35,6410 & 25,00 & 5995 & 21,3675 & 23,9316 \\
\hline $31-40$ & 32,10 & 27,4358 & 15,00 & 4672 & 22,8205 & 36,7521 \\
\hline $41-50$ & 26,80 & 22,9059 & 12,00 & 4477 & 10,2564 & 47,0085 \\
\hline $51-60$ & 22,70 & 19,4017 & 7,00 & 3083 & 5,9829 & 52,9914 \\
\hline $61-70$ & 13,60 & 11,6239 & 3,00 & 2205 & 2,5641 & 55,5555 \\
\hline $71-80$ & 6,80 & 5,8119 & 2,00 & 2941 & 1,7094 & 57,2649 \\
\hline \multicolumn{7}{|c|}{ Femelies G. austeni nourries sur lapin } \\
\hline 0 & 308,00 & 100,00 & & & & \\
\hline $1-10$ & 273,00 & $88,6.363$ & & & & \\
\hline $11-20$ & 265,50 & 86,2012 & 118,00 & 0,4444 & 38,3116 & 38,3116 \\
\hline $21-30$ & 249,60 & 81,0389 & 206,00 & 8253 & 66,8831 & 105,1948 \\
\hline $31-40$ & 244,00 & 79,2207 & 206,00 & 8442 & 66,8831 & $1.72,0779$ \\
\hline $41-50$ & 228,60 & 74,2207 & 209,00 & 9142 & 67.8571 & 239,9350 \\
\hline $51-60$ & 204,00 & 66,2337 & 161,00 & 7892 & 52,2727 & 292,2077 \\
\hline $61-70$ & 186,60 & 60,5844 & 142,00 & 7609 & 46,1038 & 338,3116 \\
\hline $71-80$ & 164,00 & 53,2467 & 130,00 & 7926 & 42,2077 & 380,5194 \\
\hline
\end{tabular}

1 = Nombre de femelles vivantes (moyenne par décade);

2 = Pourcentage de femelles vivantes, par décade;

3 = Nombre de pupes produjtes, par décade;

4 = Nombre de pupes par femelle;

$5=$ Nombre de pupes pour 100 femelles initiales, par dêcade;

$6=$ Nombre de pupes pour 100 femelles initiales (totaux cumulés).

\section{Membrane RTVS 7037 de $20 / 100$ de $\mathrm{mm}$ d'épaisseur}

Les essais effectués avec cette membrane ont porté sur deux lots de 120 femelles de G. morsitans morsitans, l'un nourri sur sang défibriné, l'autre sur sang citraté glucosé, à raison de 3,3 $\mathrm{mg}$ de glucose par millilitre de sang.

Ces deux lots ont été comparés au lot témoin de 339 femelles de $G$. morsitans nourries sur lapin et au lot de 120 femelles de G. morsitans nourries sur sang défibriné à travers la membrane RTVS 7037 de 10/100 de mm.

\section{Longévité des femelles}

La durée moyenne de vie des femelles nourries sur membrane $20 / 100$ et sang défibriné est d'environ 34 jours. Celle des femelles nourries sur membrane $20 / 100$ et sang citraté glucosé est de 41 jours.
La longévité des femelles témoins est significativement meilleure que celle des femelles nourries sur sang défibriné, quelle que soit l'épaisseur de la membrane. Il n'y a, par contre, pas de différence significative entre la longévité des femelles témoins et celle des femelles nourries sur sang citraté glucosé (tableau VII).

\section{Production de pupes}

Les productions de pupes, pour 100 femelles initiales, en 80 jours, ont été les suivantes :

-65 pupes chez G. morsitans nourrie sur membrane $20 / 100$ et sang défibriné;

- 139 pupes chez G. morsitans nourrie sur membrane 20/100 et sang citraté glucosé.

Les comparaisons des productions moyennes pour 100 femelles vivantes, par décade, révèlent une différence significative entre les témoins et les femelles nourries sur membrane 20/100 et sang défibriné, alors qu'il n'y en n'a pas 
TABLEAU $\mathrm{N}^{\circ} \mathrm{IV}$

Longêvité et production de pupes

\begin{tabular}{|c|c|c|c|c|c|c|}
\hline \multicolumn{7}{|c|}{ Lot $\mathrm{n}^{0} 1$ - Femelies G. morsitans nourries sur lapin (Témoins) } \\
\hline Jours & 1 & 2 & 3 & 4 & 5 & 6 \\
\hline $\begin{array}{r}1 \\
1-10 \\
11-20 \\
21-30 \\
31-40 \\
41-50 \\
51-60 \\
61-70 \\
71-80\end{array}$ & $\begin{array}{l}339,00 \\
324,50 \\
306,50 \\
265,00 \\
240,10 \\
221,50 \\
202,70 \\
178,00 \\
150,70\end{array}$ & $\begin{array}{l}100,00 \\
95,7227 \\
90,4129 \\
78,4660 \\
70,8259 \\
65,3392 \\
59,7935 \\
52,5073 \\
44,4542\end{array}$ & $\begin{array}{r}85,00 \\
24 B, 00 \\
213,00 \\
194,00 \\
172,00 \\
138,00 \\
106,00\end{array}$ & $\begin{array}{r}0,2773 \\
9323 \\
8871 \\
8758 \\
8485 \\
7752 \\
7033\end{array}$ & $\begin{array}{l}25,0737 \\
73,1563 \\
62,8318 \\
57,2271 \\
50,7374 \\
40,7079 \\
31,2684\end{array}$ & $\begin{array}{r}25,0737 \\
98,2300 \\
161,0619 \\
218,2890 \\
269,0265 \\
309,7345 \\
341,0029\end{array}$ \\
\hline \multicolumn{7}{|c|}{$\begin{array}{c}\text { Lot } n^{\circ} 2 \text { - Feme1les G. morsitans nourries sur membrane } 10 / 100 \\
\text { et sang défibriné }\end{array}$} \\
\hline $\begin{array}{r}1-0 \\
1-10 \\
11-20 \\
21-30 \\
31-40 \\
41-50 \\
51-60 \\
61-70 \\
71-80\end{array}$ & $\begin{array}{r}120,00 \\
113,40 \\
93,60 \\
62,40 \\
41,00 \\
30,60 \\
13,00 \\
7,60 \\
1,90\end{array}$ & $\begin{array}{r}100,00 \\
94,5000 \\
81,3333 \\
52,0000 \\
34,1666 \\
25,5000 \\
10,8333 \\
6,3333 \\
1,5833\end{array}$ & $\begin{array}{r}15,00 \\
34,00 \\
23,00 \\
21,00 \\
8,00 \\
5,00 \\
2,00\end{array}$ & $\begin{array}{r}0,1536 \\
5448 \\
5609 \\
6862 \\
6153 \\
6578 \\
1,0526\end{array}$ & $\begin{array}{r}12,5000 \\
28,3333 \\
19,1666 \\
17,5000 \\
6,6666 \\
4,1666 \\
1,6666\end{array}$ & $\begin{array}{l}12,5000 \\
40,8333 \\
60,0000 \\
77,5000 \\
84,1666 \\
88,3333 \\
90,0000\end{array}$ \\
\hline \multicolumn{7}{|c|}{$\begin{array}{l}\text { Lot } n^{*} 3-\text { Femelles de } G \text {. morsitans nourries sur membrane } 20 / 100 \\
\text { et sang défibriné }\end{array}$} \\
\hline $\begin{array}{r}1-0 \\
1-10 \\
11-20 \\
21-30 \\
31-40 \\
41-50 \\
51-60 \\
61-70 \\
71-80\end{array}$ & $\begin{array}{r}120,00 \\
106,60 \\
93,30 \\
70,90 \\
47,70 \\
26,00 \\
11,10 \\
7,80 \\
6,00\end{array}$ & $\begin{array}{r}88,8333 \\
77,7500 \\
59,0833 \\
39,7500 \\
21,6666 \\
9,2500 \\
6,5000 \\
5,0000\end{array}$ & $\begin{array}{r}0,00 \\
48,00 \\
16,00 \\
5,00 \\
2,00 \\
4,00 \\
3,00\end{array}$ & $\begin{array}{r}0,0000 \\
6770 \\
3354 \\
1923 \\
1801 \\
512 \mathrm{~B} \\
5000\end{array}$ & $\begin{array}{r}0,0000 \\
40,0000 \\
13,3333 \\
4,2666 \\
1,6666 \\
3,3333 \\
2,5000\end{array}$ & $\begin{array}{r}0000 \\
40,0000 \\
53,3333 \\
57,4999 \\
59,1666 \\
62,4999 \\
64,9999\end{array}$ \\
\hline \multicolumn{7}{|c|}{$\begin{array}{c}\text { Lot } n^{0} 4 \text { - Femelles de } G \text {. morsitans nourries sur membrane 20/100 } \\
\text { et sang citraté glucosé }\end{array}$} \\
\hline $\begin{array}{r}0 \\
1-10 \\
11-20 \\
21-30 \\
31-40 \\
41-50 \\
51-60 \\
61-70 \\
71-80\end{array}$ & $\begin{array}{r}120,00 \\
111,20 \\
93,00 \\
78,10 \\
61,40 \\
49,60 \\
27,50 \\
16,00 \\
8,00\end{array}$ & $\begin{array}{r}92,6666 \\
77,5000 \\
65,0833 \\
51,1666 \\
41,3333 \\
22,9166 \\
13,3333 \\
6,6666\end{array}$ & $\begin{array}{r}15,00 \\
46,00 \\
34,00 \\
46,00 \\
12,00 \\
8,00 \\
6,00\end{array}$ & $\begin{array}{r}0,1612 \\
5889 \\
5537 \\
9274 \\
4363 \\
5000 \\
7500\end{array}$ & $\begin{array}{r}12,5000 \\
38,3333 \\
28,3333 \\
38,3333 \\
10,0000 \\
6,6666 \\
5,0000\end{array}$ & $\begin{array}{r}12,5000 \\
50,8333 \\
79,1666 \\
117,4999 \\
127,4999 \\
134,1666 \\
139,1666\end{array}$ \\
\hline
\end{tabular}

1 = Nombre de femelles vivantes (moyenne par dêcade); 4 = Nombre de pupes par femelle;

2 = Pourcentage de femelles vivantes, par décade; $\quad 5$ = Nombre de pupes pour 100 femelles initiales

3 = Nombre de pupes produites par décade; par décade:

$6=$ Nombre de pupes pour 100 femelles initiales (totaux cumulés).

entre les témoins et les femelles nourries sur membrane $10 / 100$ et sang défibriné, ou sur membrane $20 / 100$ et sang citraté glucosé (tableau VIII). La nourriture sur sang citraté glucosé compense donc l'action défavorable due à la trop grande épaisseur de la membrane.

\section{Poids des pupes}

Les pupes produites par les femelles nourries sur membrane $20 / 100$ et sang défibriné ont un poids moyen de $23,72 \mathrm{mg} \pm 0,70$. Ce poids n'est pas significativement différent de celui des pupes produites par les femelles nourries sur membrane $10 / 100$ et sang défibriné (tableau IX).

Les pupes issues des femelles nourries sur membrane $20 / 100$ et sang citraté glucosé ont un poids moyen de $24,77 \mathrm{mg} \pm 0,45$. Il est significativement plus élevé que celui des pupes produites par les femelles nourries sur sang 
TABLEAU $\mathrm{N}^{\circ} \mathrm{V}$

Production de pupes pour 100 femelles, par décade, en 100 jours.

\begin{tabular}{|c|c|c|c|c|c|}
\hline $\mathrm{N}^{\bullet}$ & Caractéristique & $\begin{array}{c}\text { Productions } \\
\text { moyennes de } \\
\text { pupes pour } 100 \\
\text { femelles (m) }\end{array}$ & $\begin{array}{c}\text { Ecart-type } \\
(s)\end{array}$ & $\begin{array}{l}\text { Coefficient de } \\
\text { variation }(p, 100) \\
(100 \mathrm{~s} / \mathrm{m})\end{array}$ & $\begin{array}{c}\text { Intervalle de confiance } \\
\text { de la moyenne } \\
\text { théorique pour } \\
\alpha=5 \text { p.100 }\end{array}$ \\
\hline 1 & $\begin{array}{l}\text { G. austeni femelles } \\
\text { nourries sur lapin }\end{array}$ & 69,74 & 28,1128 & 40,3090 & $52,31-87,16$ \\
\hline 2 & $\begin{array}{l}\text { G. austeni femelles } \\
\text { nourries sur membrane } \\
10 / 100\end{array}$ & 21,98 & 20,3287 & 92,4538 & $9,38-34,58$ \\
\hline 3 & $\begin{array}{l}\text { G. morsitans femelles } \\
\text { nourries sur lapin }\end{array}$ & 63,73 & 29,3516 & 46,0542 & $45,54-81,92$ \\
\hline 4 & $\begin{array}{l}\text { G. morsitans femelles } \\
\text { nourries sur membrane } \\
10 / 100\end{array}$ & 48,81 & 35,6270 & 72,9871 & $26,73-70,89$ \\
\hline \multicolumn{3}{|c|}{ Comparaison des moyennes entre : } & Valeur de $t$ & $\begin{array}{l}\text { Degré de } \\
\text { liberté }\end{array}$ & $\begin{array}{l}\text { Signification poux } \\
\alpha=5 p \cdot 100\end{array}$ \\
\hline \multicolumn{3}{|c|}{$\begin{array}{c}1 \text { et } 2 \text { (G. austeni nourries sur lapin et } \\
\text { sur membrane } 10 / 100 \text { ) }\end{array}$} & 1,8657 & 18 & N.S. \\
\hline \multicolumn{3}{|c|}{$\begin{array}{c}\text { 3 et } 4 \text { (G. moraitans nourries sur lapin et } \\
\text { sur membrane } 10 / 100)\end{array}$} & 0,5094 & 18 & N.S. \\
\hline \multicolumn{3}{|c|}{$\begin{array}{c}1 \text { et } 3 \text { (G. austeni et } G \text {. monsitans nourries } \\
\text { gur lapin) }\end{array}$} & 0,1848 & 18 & N.S. \\
\hline \multicolumn{3}{|c|}{$\begin{array}{c}2 \text { et } 4 \text { (G. austèni et } G \text {, morsitans nourries } \\
\text { sur membrane } 10 / 100 \text { ) }\end{array}$} & 1,2577 & 18 & N.S. \\
\hline \multicolumn{3}{|c|}{$\begin{array}{l}2 \text { et } 3 \text { (G. austeni nourries sur membrane } \\
\text { 10/100, G. morsitans nourries sur lapin) }\end{array}$} & 1,7303 & 18 & N.S. \\
\hline \multicolumn{3}{|c|}{$\begin{array}{l}1 \text { et } 4 \text { (G. austeni nourries sur lapin } \\
\text { G. morsitans nourries sur membrane } \\
10 / 100)\end{array}$} & 0,6861 & 18 & N.S. \\
\hline
\end{tabular}

TABLEAU $\mathrm{N}^{\bullet} \mathrm{VI}$

Poids des pupes produites

\begin{tabular}{|c|c|c|c|c|c|c|}
\hline $\mathrm{N}^{\bullet}$ & Caractêristique & $\begin{array}{l}\text { Nombre de } \\
\text { pupes }(n)\end{array}$ & $\begin{array}{l}\text { Poids moyen } \\
\text { en mg (m) }\end{array}$ & $\begin{array}{c}\text { Ecart-type } \\
(s)\end{array}$ & $\begin{array}{l}\text { Coefficient de } \\
\text { variation }(p, 100) \\
(100 \mathrm{~s} / \mathrm{m})\end{array}$ & $\begin{array}{c}\text { Intervalle de confiance } \\
\text { de la moyenne } \\
\text { théorique pour } \\
\dot{\nu}=5 \mathrm{p} .100\end{array}$ \\
\hline 1 & $\begin{array}{l}\text { G. austeni femelles } \\
\text { nourrieg sur lapin }\end{array}$ & 100 & 25,47 & 3,7422 & 14,6924 & $24,73-26,20$ \\
\hline 2 & $\begin{array}{l}\text { G. austeni femelles } \\
\text { nourries sur membrane } \\
10 / 100\end{array}$ & 67 & 16,47 & 2,9052 & 17,6372 & $15,77-17,16$ \\
\hline 3 & $\begin{array}{l}\text { G. morsitans femelles } \\
\text { nourries sur lapin }\end{array}$ & B8 & 27,73 & 4,1312 & 14,8964 & $26,87-28,59$ \\
\hline 4 & $\begin{array}{l}\text { G. morsitans femelles } \\
\text { nourries sur membrane } \\
10 / 100\end{array}$ & 95 & 23,59 & 3,0359 & 12,8674 & $22,98-24,20$ \\
\hline \multicolumn{4}{|c|}{ Comparaison des poids moyens entre : } & \multicolumn{2}{|c|}{ Valeur de $\varepsilon$} & $\begin{array}{c}\text { Signification pour } \\
\alpha=5 p .100\end{array}$ \\
\hline \multicolumn{4}{|c|}{$\begin{array}{l}1 \text { et } 2 \text { Pupes produites par les femelles de } \\
G \text {, austeni nourries sur lapin et sur } \\
\text { membrane } 10 / 100\end{array}$} & \multicolumn{2}{|r|}{17,4458} & T.S. \\
\hline \multicolumn{4}{|c|}{$\begin{array}{l}3 \text { et } 4 \text { Pupes produices par les femelles de } \\
\text { G. morsitans nourries sur lapin et sur } \\
\text { membrane } 10 / 100\end{array}$} & \multicolumn{2}{|r|}{7,6735} & T.S. \\
\hline
\end{tabular}




\section{TABLEAU N"VII}

Longévité des femelles parentes (G. morsitans)

\begin{tabular}{|c|c|c|}
\hline Comparaison du nombre de $q$ vivantes (en p.100) entre : & Valeur de $E$ & Signification \\
\hline $\begin{array}{ll}1 \text { et } 2 \text { : Témoins et memb. } & 20 / 100+\text { sang défibriné } \\
1 \text { et } 3 \text { : Témoins et memb. } & 20 / 100+\text { sang défibrinë } \\
1 \text { et } 4 \text { : Témoins et memb. } & 20 / 100+\text { sang citraté glucosé }\end{array}$ & $\begin{array}{l}2,2682 \\
2,3334 \\
1,8462\end{array}$ & 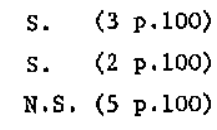 \\
\hline
\end{tabular}

Conclusions - 1) Longévitê đes femelles têmoins significativement meilleure que celle des femelles nourries sur sang déffbriné, quelle que solt l'épalsseur de la membrane.

2) Pag de différence significative entre les longêvitếs des femelles tếmoins et des femelles nourries sur sang cltraté glucosê.

\section{TABLEAU N"VIII}

Production de pupes (G. morsitans)

\begin{tabular}{|c|c|c|}
\hline Comparaison du nombre moyen de pupes par femelle entre : & Valeur de $E$ & Signification \\
\hline $\begin{array}{l}\text { 1' et } 2 \text { - Témoins et memb. } 10 / 100+\text { sang défibriné } \\
1 \text { et } 3 \text { : Témoins et memb. } 20 / 100+\text { sang défibriné } \\
1 \text { et } 4 \text { : Têmoins et memb. } 20 / 100 \text { + sang citraté glucosé }\end{array}$ & $\begin{array}{l}1,1200 \\
3,3702 \\
1,5829\end{array}$ & $\begin{array}{l}\text { N.S. }(5 \text { p.100) } \\
\text { S. } \quad(1 \mathrm{p} .1000) \\
\text { N.S. }(5 \mathrm{p} .100)\end{array}$ \\
\hline
\end{tabular}

Conclusions - 1) Les femelles nourrles sur membrane épaisse (20/100) et gang défibriné ont un très faible taux de production.

2) La nourriture sur sang citraté glucosé compense l'action défavorable due à la trop grande ếpaisseur de la membrane.

TABLEAU $\mathrm{N}^{\bullet} \mathrm{IX}$

Poids des pupes $F_{1}$

\begin{tabular}{|c|c|c|c|c|c|}
\hline $\mathrm{N}^{\bullet}$ & Caractéristique & $\begin{array}{l}\text { Nombre } \\
\text { de pupes } \\
\text { (n) }\end{array}$ & $\begin{array}{c}\text { Poids } \\
\text { moyen } \\
(\mathrm{mg})(\mathrm{m})\end{array}$ & $\begin{array}{l}\text { Ecart- } \\
\text { type } \\
(s)\end{array}$ & $\begin{array}{c}\text { Intervalle de confiance } \\
\text { de la moyenne } \\
\text { théorique pour } \\
\alpha=5 \text { p.100 }\end{array}$ \\
\hline 1 & G. morsitans, femelles nourries sur lapin (Témoins) & 88 & 27,73 & 4,1312 & $26,87-28,59$ \\
\hline 2 & G. morgitans, membrane $10 / 100$ et sang défibrinê & 102 & 23,65 & 3,2144 & $23,02-24,27$ \\
\hline 3 & G. morsitans, membrane $20 / 100$ et sang défibriné & Bo & 23,72 & 3,1948 & $23,02-24,42$ \\
\hline 4 & $\begin{array}{l}\text { G. morsitans, membrane } 20 / 100 \text { et sang citraté } \\
\text { glucosé }\end{array}$ & 167 & 24,77 & 3,0101 & $24,32-25,23^{t}$ \\
\hline & Comparaison des poids moyens entre : & \multicolumn{2}{|c|}{ Valeur de $\epsilon$} & \multicolumn{2}{|r|}{ Signification } \\
\hline & et 2 : Témoins et memb. $10 / 100+$ sang défibrinĕ & \multicolumn{2}{|r|}{7,5112} & \multicolumn{2}{|r|}{ T.S. $(1 \mathrm{p} .1000)$} \\
\hline & et 3 : Témoins et memb. $20 / 100$ + sang dëfibriné & \multicolumn{2}{|r|}{7,0724} & \multicolumn{2}{|r|}{ T.S. (1 p.1000) } \\
\hline & et 4 : Témoins et memb. $20 / 100$ + sang citraté glucosé & \multicolumn{2}{|r|}{5,9344} & \multicolumn{2}{|r|}{ T.S. (1 p.1000) } \\
\hline & $\begin{array}{l}3 \text { : Memb. } 10 / 100+\text { sang défibrinë et memb. } \\
20 / 100 \text { + sang dëfibriné }\end{array}$ & \multicolumn{2}{|r|}{0,1484} & \multicolumn{2}{|r|}{ N.s. $(5$ p.100) } \\
\hline & $\begin{array}{l}4: \text { Memb. } 10 / 100+\text { sang défibriné et memb. } \\
20 / 100 \text { + sang citraté glucosé }\end{array}$ & \multicolumn{2}{|r|}{2,8519} & \multicolumn{2}{|r|}{ s. $\quad(1$ p.100) } \\
\hline \multicolumn{2}{|r|}{$\begin{array}{c}3 \text { et } 4: \text { Memb. } 20 / 100+\text { sang défibrinẻ et memb. } \\
20 / 100+\text { sang citraté glucosé. }\end{array}$} & \multicolumn{2}{|r|}{2,4711} & \multicolumn{2}{|r|}{ s. (2 p. 100$)$} \\
\hline
\end{tabular}

Conclusions - 1) Poids des pupes produites par les témoins très gignificativement plus ėlevé que celui des pupes produites par tous les lots d'expérience.

2) Poids des pupes produites par les femelles nourries sur sang citraté glucosẻ significativement plus élevé que celui des pupes produites par les femelles nourries sur sang dêfibrinê. 
TABLEAU $\mathrm{N}^{\circ} \mathrm{X}$

Poids des pupes $F_{2}$

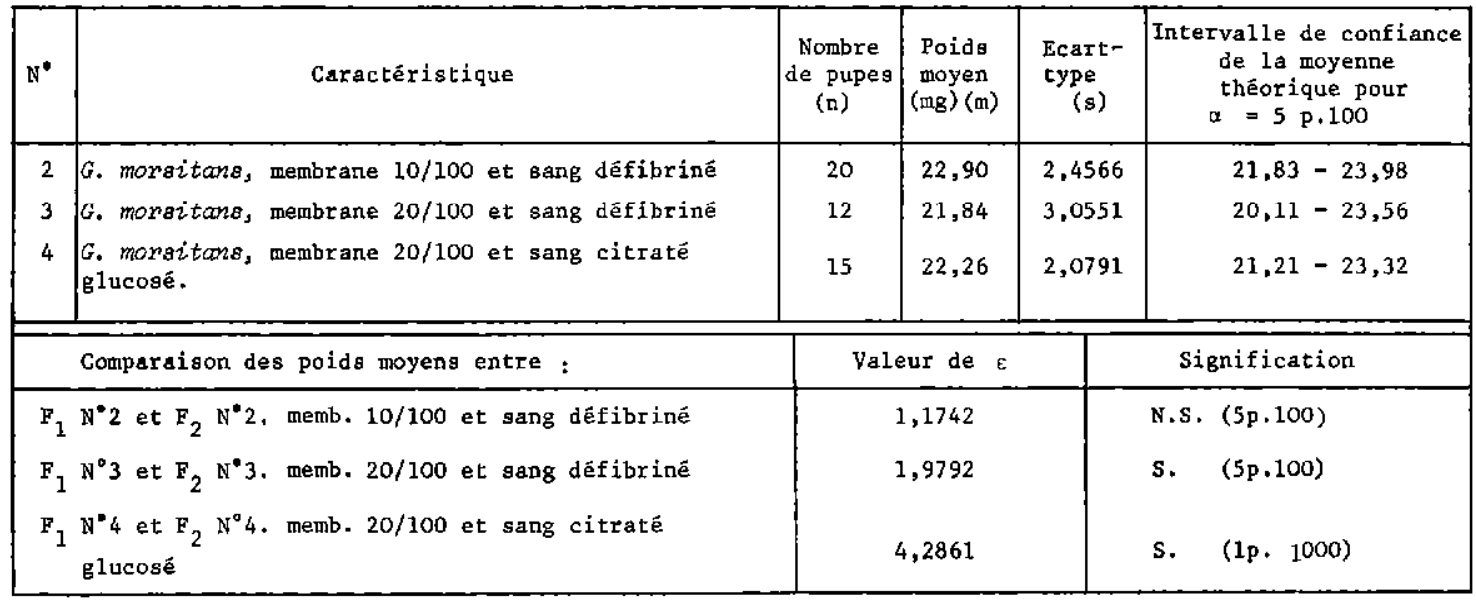

Conclusions - 1) Pas de variation de poids entre les pupes produites par les femelles parentes nourries sur membrane 10/100 + sang défibrinè et celles produites par leurs filles.

2) Forte ou trè forte chute de poids des pupes produices par les femelles de lère génération, nourries sur membrane 20/100 et sang dêfibriné ou sang citraté glucosé, par rapport au poids des pupes produites par leurs mères.

défibriné, quelle que soit l'épaisseur de la membrane, mais inférieur à celui des pupes produites par les femelles témoins $(27,33 \mathrm{mg} \pm$ 0,86 ).

\section{Descendants de première génération des femelles de $G$. morsitans}

Les adultes éclos des pupes produites par les femelles de $G$. morsitans nourries sur membrane $10 / 100$ ou 20/100 ont été nourris dans les mêmes conditions que leurs parents et ont fait l'objet d'une étude analogue.

Les résultats obtenus avec ces descendants de $1^{\text {re }}$ génération se résument ainsi :

\section{Nourriture sur membrane $10 / 100$ et sang défibriné}

- Longévité moyenne des mâles $=19,6$ jours.

- Longévité moyenne des femelles = 27,9 jours.

- Pourcentage des femelles reproductrices (ayant vécu au moins 20 jours) $=51,02 \mathrm{p}$. 100.

- Nombre de pupes produites par femelle reproductrice, en 60 jours $=0,80$.

- Poids moyen des pupes $F_{2}=22,90 \pm$ $1,07 \mathrm{mg}$.
Nourriture sur membrane 20/100

et sang défibriné

- Longévité moyenne des mâles $=21,4$ jours.

- Longévité moyenne des femelles = 28,3 jours.

- Pourcentage de femelles reproductrices $=$ 64,5 p. 100.

- Nombre de pupes produites par femelle reproductrice, en 60 jours $=0,50$.

- Poids moyen des pupes $\mathbf{F}_{2}=21,84 \pm$ $1,72 \mathrm{mg}$.

Nourriture sur membrane $20 / 100$

et sang citraté glucosé

- Longévité moyenne des mâles = 15,0 jours.

- Longévité moyenne des femelles $=$ 19,5 jours.

- Pourcentage de femelles reproductrices $=$ 37,6 p. 100.

- Nombre de pupes produites par femelle reproductrice, en 60 jours $=0,46$.

- Poids moyen de pupes $F_{2}=22,26 \pm$ $1,05 \mathrm{mg}$. 


\section{CONCLUSIONS}

Cette première étude a montré qu'il était possible de nourrir des glossines, de façon artificielle, sans avoir recours à un animal vivant. Si les résultats obtenus ne sont pas parfaits (longévité réduite, production de pupes inférieure à celle des témoins, faible poids des pupes produites), il apparaît que ces résultats tiennent davantage à la composition même du liquide nutritif qui est fourni aux glossines (sang citraté ou sang défibriné) qu'à la composition de la membrane.

A condition que leur épaisseur ne soit pas trop importante, les membranes de silicone sont aisément traversées par l'appareil piqueur des glossines. L'élasticité de ce type de membrane lui permet de se refermer après le retrait du proboscis. Ces membranes sont chimiquement inertes et les qualités physiques des silicones permettent de les stériliser à des températures élevées et de les conserver indéfiniment.

A la suite des premiers essais effectués, il est apparu que les meilleurs résultats ont été obtenus avec la membrane RTVS 7037 de 10/100 de mm d'épaisseur, d'une part, et avec le sang citraté glucosé, d'autre part.

Les principaux points que cette série d'expérimentations a permis de faire ressortir, peuvent se résumer ainsi :

1. La nourriture sur membrane diminue dans tous les cas la longévité des femelles, bien que les femelles nourries sur sang citraté glucosé aient une meilleure longévité que les femelles nourries sur sang défibriné;

2. L'épaisseur de la membrane influe sur la capacité reproductrice des femelles. Lorsque cette épaisseur atteint $20 / 100$ de $\mathrm{mm}$, les femelles éprouvent des difficultés à absorber le milieu nutritif et se nourrissent insuffisamment pour assurer le développement de la larve jusqu'à son terme. Ce défaut d'alimentation est en partie compensé par les qualités nutri- tives du milieu (sang citraté supplémenté en glucose);

3. La nourriture sur sang citraté glucosé, comparativement à la nourriture sur sang défibriné, améliore à la fois la longévité des femelles et leurs capacités reproductrices, donc le nombre total de pupes produites;

4. Le poids des pupes produites par les femelles nourries sur membrane est toujours plus faible que celui des pupes produites par les femelles témoins. Cependant, la nourriture sur sang citraté glucosé augmente de façon significative le poids de ces pupes, par rapport au poids des pupes issues de femelles nourries sur sang défibriné;

5. Chez tous les descendants de première génération nourris artificiellement, la longévité est considérablement abrégée, la mortalité est très forte chez les jeunes, la production de pupes des femelles est nettement inférieure à celle des parents. Le poids des pupes produites est identique à celui des pupes produites par les parents, ou nettement plus faible, et toujours inférieur au poids des pupes produites par les femelles témoins.

D'autres essais sont en cours, avec la membrane RTVS 7037 de $10 / 100$ et des milieux nutritifs différents (sang défibriné ou sang citraté glucosé), additionnés de divers composés: fibrinogène, acides aminés, adénosine triphosphorique, dans le but de déterminer la meilleure composition du milieu.

Nous espérons ainsi pouvoir augmenter la longévité des femelles, et par conséquent leur taux de production de pupes, qui en est étroitement dépendant, ainsi que le poids des pupes produites. Nous pourrons ensuite essayer d'entretenir plusieurs générations avec cet unique mode d'alimentation.

Ce n'est que lorsque ces différents problèmes seront résolus que l'on pourra espérer obtenir un élevage en masse de ces insectes sans utiliser d'animaux hôtes vivants.

\section{SUMMARY}

\section{A synthetic membrane used for artificial feeding of Glossinae}

Trials of artificial feeding of Glossinae through a synthetic membrane were carried out. The results obtained show that silicone membranes of $10 / 100 \mathrm{~mm}$ thickness allow these insects artificially fed. These membranes are chemically inert and silicone physical qualities allow sterilize them at high temperatures and conserve them indefinitely. The 
feeding by means of sheep citrated glucosed blood, compared with the feeding by means of defibrinated blood, improves the female longevity, their reproductive performance and the produced pupae weight, which however are lower than results obtained in control flies fed on rabbit.

\section{RESUMEN}

\section{Una membrana sintética utilizada para la alimentación artificial de las glosinas (Diptera Muscidae)}

Se efectuaron ensayos de alimentación artificial de las glosinas de través una membrana sintética. Los resultados obtenidos muestran que membranas de silicono de $10 / 100 \mathrm{~mm}$ de espesor permiten la alimentación artificial de dichos insectos. Estas membranas son quimicamente inertes y las cualidades físicas de los siliconos permiten esterilizarlos en temperaturas elevadas y conservarlos indefinidamente. La alimentación mediante sangre citratada glucosada de oveja, comparada con la mediante sangre desfibrinada, mejora la longevidad de las hembras, su capacidad de reproducción y el peso de las pupas producidas, que sin embargo son inferiores a los resultados obtenidos en moscas-testigos alimentadas sobre conejo.

\section{BIBLIOGRAPHIE}

1. AZEVEDO (J. F.), PINHAO (R. C.) et SANTOS (A.M. T.). Artificial breeding of tsetse fly. I. Factors connected with the feeding membrane and blood meals type. An. Esc. nac. Saude publ. Med. trop., 1968, 2 (1-4) : 11-17.

2. AZEVEDO (J.F.), SANTOS (A.M.T.) et PINHAO (R. C.). New data on the artificial breeding of Glossina morsitans. 1er Symposium sur l'élevage en laborasoire des Glossines, Lisbonne, 1970.

3. COCKINGS (K. L.). Laboratory maintenance of Glossina pallidipes. Rapport E.A.T.R.O., 1969, 14-18.

4. GALUN (R.), Recent development in the biochemistry and feeding behaviour of haematophagous arthropods as applied to their mass rearing. Symposium on the sterility principle for insect control or eradication I.A.E.A./F.A.O. Athènes 14-18 sept. 1970.

5. GALUN (R.) et MARGALIT (J.). Artificial feeding stimuli of the tsetse fly Glossina austeni. 1er Symposium sur l'élevage en laboratoire des Glossines, Lisbonne, 1970.

6. LANGLEY (P. A.) et MALY (H.). Membrane feeding technique for tsetse flies (Glossina spp.). Nature, 1969, 221 (5183): 855-856.

7. Mc MAHON (J.P.). Artificial feeding of Simulium vectors of human and bovine onchocerciasis. Bull, Org. mond. Santé, 1968, 38: 957-966.

8. MEWS (A. R.) et RUHM (F.). Maintenance of $G$. morsitans West. fed through an artificial membrane on defibrinated blood. Symposium on the sterility principle for insect control or eradication I.A.E.A.FF.A.O. Athènes, 14-18 sept. 1970. 